\title{
Temperature effect of muon component and practical questions of how to take into account in real time
}

\author{
M. Berkova ${ }^{1,2}$, A. Belov ${ }^{1}$, E. Eroshenko ${ }^{1}$, and V. Yanke $^{1}$ \\ ${ }^{1}$ Institute of Terrestrial Magnetism, Ionosphere and Radio Wave Propagation RAS (IZMIRAN), Moscow, Russia \\ ${ }^{2}$ Institute of Applied Mechanics RAS (IAM RAS), Moscow, Russia \\ Correspondence to: V. Yanke (yanke@izmiran.ru)
}

Received: 26 October 2010 - Revised: 2 September 2012 - Accepted: 11 October 2012 - Published: 21 December 2012

\begin{abstract}
A method has been developed to correct in realtime the cosmic ray $(\mathrm{CR})$ muon component, observed by the muon telescopes of different geometry, for temperature effect.
\end{abstract}

\section{Introduction}

Wide use of muon detectors' data is restrained by presence of significant temperature effect inherent to the muon component of secondary cosmic rays. To exclude the temperature effect the data of aerologic soundings close to the detector location are necessary. Aerologic soundings should be carried out regularly and with proper time resolution. But more often such data are absent in general and impossible to restore in retrospect, which results in partial data correction of the muon telescopes. Therefore it is more effective to use the results of the global atmosphere models, which make it possible to obtain the temperature profile of the atmosphere in any place and at any time. Such an approach has been realized in a number of works (e.g. Belov et al., 1995; Berkova et al., 2008; Tilav et al., 2009; Adamson et al., 2010). These models provide a way to allow for the temperature effect in real time.

One of the goals of this work is the development of mathematical tools and software for the queries of a model's data and for the temperature effect exclusion in real time. Another goal is a comparison of different methods of the temperature effect exclusion: integral method and alternative approximate ones.

\section{Temperature effect of the muon component}

Temperature effect of the CR muon component is caused by decays of pions and muons and their interactions with atmospheric nuclei. At the energies typical for the ground-based detectors, the negative temperature effect of the CR muon component prevails; at the energies typical for the underground detectors, the positive temperature effect prevails.

To account these temperature effects, the integral method was developed. It is presented in detail in Maeda and Wada (1954) and Dorman (1972). Temperature variations are determined as

$\delta I_{T}=\int_{0}^{h_{0}} \alpha(h) \cdot \delta T(h) \cdot d h$,

where $\delta I_{T}$ represents temperature variations of CR intensity, $\delta T(h)$ represents temperature variations from the base period, and therefore $\delta T(h)=T_{B}(h)-T(h)$. Densities of the temperature coefficients $\alpha(h)$ for different detectors in this work are the same as in the work of Berkova et al. (2008).

In addition there are two alternative empirical methods. The method of effective level of generation is based on the assumption that muons are generally generated at the isobaric level, usually taken for $100 \mathrm{mb}$, their height varying with the change in atmosphere temperature. According to Blacket (1938) and Duperier (1944), the variation of the muon component intensity is correlated with the height of generation level $\delta H$ and with the air temperature in this layer $\delta T$, i.e.

$\delta I_{T}=\alpha_{H} \delta H+\alpha_{T} \delta T$,

where $\alpha_{H}(\% / \mathrm{km})$ is so-called decay factor - the negative effect - and $\alpha_{T}\left(\% /{ }^{\circ} \mathrm{C}\right.$ - the positive temperature coefficient.

The method of mass-average temperature is based on the determination of the atmosphere mass-average temperature. As the densities of temperature coefficient $\alpha(h)$ for the ground-based detectors are not strongly changed with the atmospheric depth $h$, the average $\bar{\alpha}(h)$ can be put out behind the integral sign Eq. (1) as follows:

$\delta I_{T}=\bar{\alpha} \int_{0}^{h_{0}} \delta T(h) \cdot d h=\bar{\alpha} \sum_{n=1}^{\text {Lskin }} \cdot \frac{\Delta h_{n}}{h_{0}} \cdot \overline{T_{n}}=\bar{\alpha} \cdot \delta T_{m}$, 


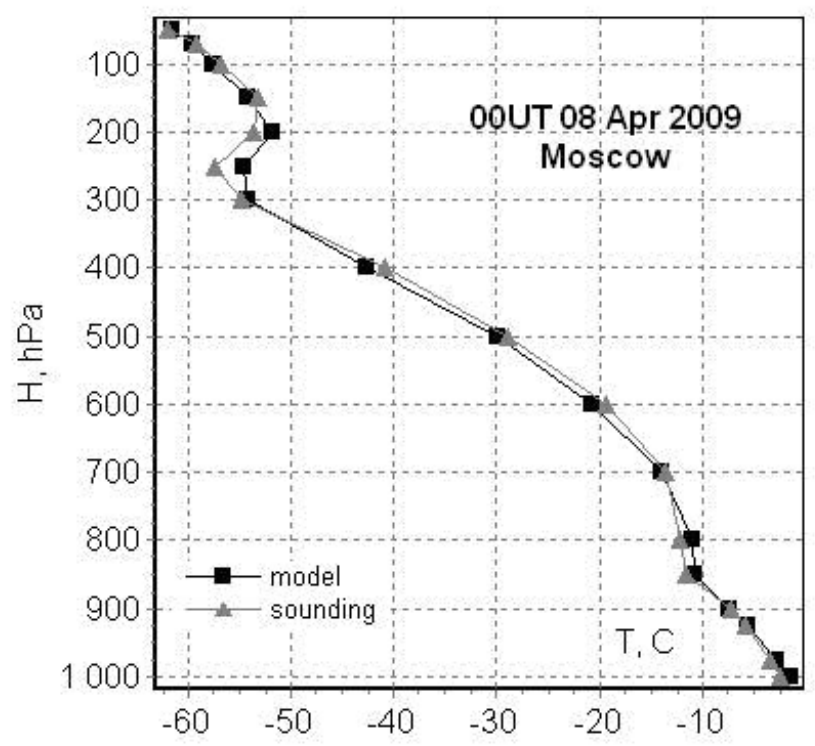

Fig. 1. Vertical temperature distribution in atmosphere for Moscow: model (black squares) and measured (grey triangles).

where $T_{m}$ is mass-average temperature and $L_{\text {skin }}$ is surface layer number. This method was first noted by Dvornikov et al. (1976), and has been used in a number of works (Yanchukovsky et al., 2007; Tilav et al., 2009; Adamson et al., 2010). All these methods require data of the vertical atmosphere temperature profile.

\section{Temperature data}

In meteorology on the base of generalized meteorological data, models are built to enable obtaining the temperature profile of the atmosphere. In this work the temperature model data of the Global Forecast System (GFS) represented by the NCEP (National Centers for Environmental Prediction USA) have been used (http://www.nco.ncep.noaa.gov/pmb/ products/gfs/). The GFS model makes it possible to obtain both retrospective and prognostic data of a 3-D temperature field. The model's outputs are temperature data at 17 standard isobaric levels, four times a day (00:00, 06:00, 12:00, 18:00 UT). The data are interpolated on the grid of $1^{\circ} \times 1^{\circ}$ resolution. To obtain hourly data, interpolation by the cubic spline function on nine nodal points is carried out (Forsythe et al., 1980). We verified the GFS model's data for Moscow for 1999. For this purpose we compared the model's data with the sounding data. The results for 8 April 2009 are depicted in Fig. 1, where temperature distribution from the GFS model is marked by black squares and experimental data are marked by grey triangles. The accuracy of such data is about several degrees depending on isobaric level. Most error is at the observation level, as the temperature is more changeable

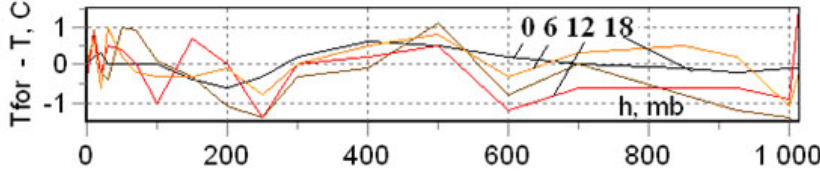

Fig. 2. Discrepancy between forecast and updated data at 00:00, 06:00, 12:00 and 18:00 UT.

in the lower layer. So, for the observation level we always use the temperature measured in situ.

Real-time processing is providing by the current day forecast. Every day at 00:00 UT we get a temperature forecast for the current day, and use this data for real-time processing. Next day the GFS model updates its forecast with the real assimilated data. We get updated data as well and correct our result for the previous day, which was calculated in real-time mode by the forecast temperature data.

As is seen in Fig. 2, a discrepancy between daily forecasted and updated data sets is about $1^{\circ}$ or less within required accuracy.

\section{Data of continuous cosmic ray monitoring}

The developed method was applied for processing the telescope's data accessible in real-time. There were two such muon detectors: scintillation super telescopes Nagoya (17 directions) and counter telescopes Moscow (15 directions).

At present, data of the muon telescopes world network are corrected for the temperature effect by the method of effective level of generation (Okazaki et al., 2008). But because of the absence of sounding temperature data, only $25 \%$ of the telescope's data were corrected by this method.

\section{Results}

Results of exclusion of the temperature variations by all the methods described above for two muon telescopes, Nagoya and Moscow, are presented in Fig. 3. Hourly variations were obtained relative to the 2009 base year. There are original uncorrected data for the vertical direction (blue curve), temperature variations obtained by the integral method (brown curve), the same by the mass-average temperature method (orange curve), and the same by the method of effective generation level (green curve, only for Nagoya). Finally, for each telescope original data were corrected by the integral method (red curve), and they are compared with variations of the equatorial neutron monitor Thailand (black curve), as its effective rigidity is the closest to effective rigidities of ground-based muon telescopes. We can conclude that the temperature effect calculated by the mass-average temperature method visually coincides with the temperature effect 

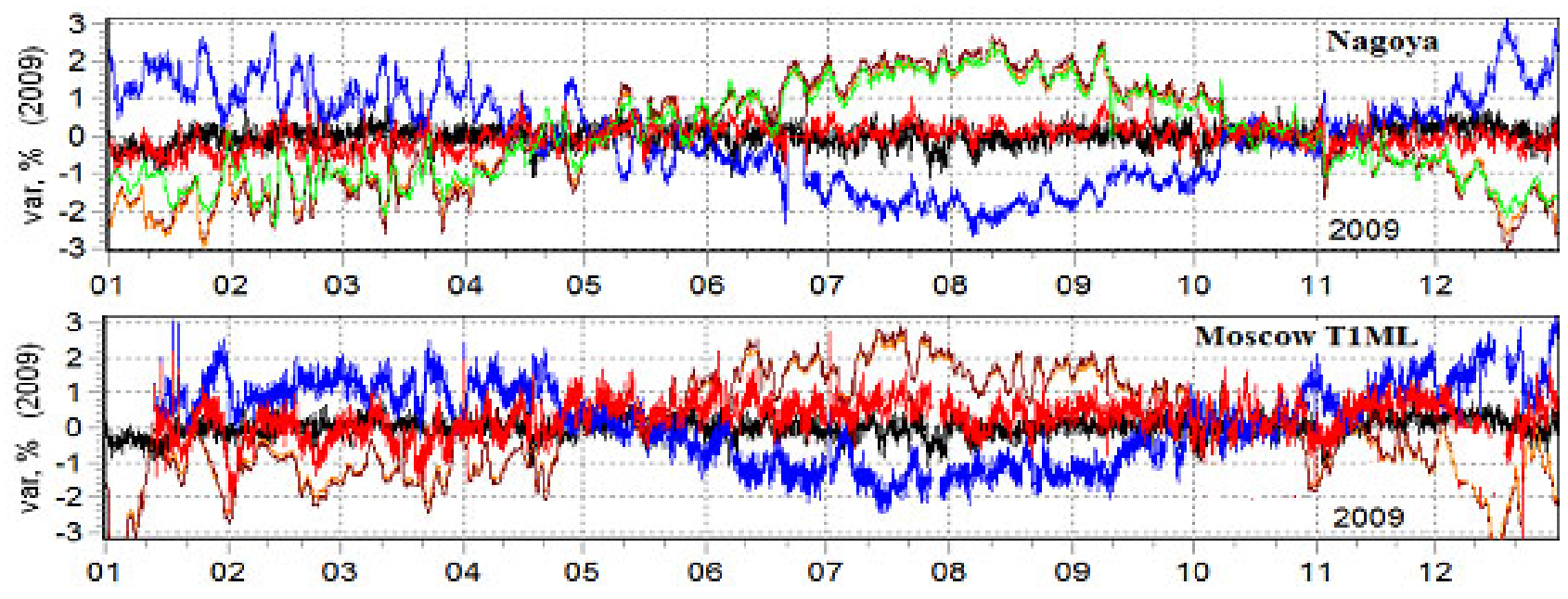

Fig. 3. Temperature variation exclusion from the Nagoya and Moscow muon telescopes data for 2009. Here: 1) original uncorrected data for the vertical direction (blue curve); 2) temperature variations obtained by the integral method (brown curve); 3 ) the same by the mass-average temperature method (orange curve); 4) the same by the method of effective generation level (green curve); 5) data corrected by the integral method (red curve); 6) variations of the equatorial neutron monitor Thailand (black curve).

calculated by the integral method. The method of effective generation level that was applied to the Nagoya data has the most discrepancy with two other methods in winter. At Nagoya, corrected data are in good agreement with variations of the Thailand neutron monitor. But results for the Moscow telescope are not in as good agreement. First of all, this is due to worse statistics as the Moscow telescope's area is 25 times less then that of Nagoya. Secondly, the Moscow telescope in 2009 registered the common ionizating component because there was no lead absorber. The presence of soft component leads to additional variations of terrestrial origin.

\section{Conclusions}

In the case of ground-based detectors, all three methods are suitable. Worse statistics from the Moscow telescope and the contribution from the common ionizating component results in worse agreement with corrected temperature effect data and with variations of the Thailand neutron monitor.

All the methods under consideration need data of the atmospheric temperature profile. The method developed using the temperature data, obtained from the GFS model, enables to correct for the temperature effect of hourly data from the muon telescopes with sufficient accuracy.

The original and corrected data of the muon detectors are available at the address ftp://cr0.izmiran.rssi.ru/ CosRay!/FTP_TEL/. The full version of this work can be accessed at http://cr0.izmiran.rssi.ru/gmdnet/Publication/ GEO_15_eng.pdf

Acknowledgements. This work is partly supported by Program No. 6 BR of the Presidium RAS "Neutrino
Physics and Astrophysics" and acknowledgements to http://cr0.izmiran.ru/ThankYou/main.htm.

Edited by: K. Scherer

Reviewed by: three anonymous referees

\section{References}

Adamson, P. and the MINOS collaboration: Observation of muon intensity variations by season with the MINOS far detector, Phys. Rev., doi:10.1103/PhysRevD.81.012001, 2010.

Belov, A. V., Dorman, L. I., Gushchina, R. T., and Yanke, V. G.: Temporal and latitude dependence of the temperature effect for neutron component of cosmic rays, in: Proceedings of the 24-th ICRC., Rome, Italy, 28 August - 8 September 1995, 4, 1141 1144, 1995.

Berkova, M., Belov, A., Eroshenko, E., and Yanke, V.: Temperature effect of the muon component of cosmic ray and practical possibilities of its accounting, in: Proceedings of the $21^{\text {st }}$ ECRS, Košice, Slovakia, 9-12 September 2008, 123 - 126, 2008.

Blackett P.H.: On the instability of the barytron and the temperature effect of cosmic rays, Phys. Rev., 54, 973-974, 1938.

Dorman, L. I.: Meteorological effects of cosmic rays: Nauka, Moscow, USSR, 1972.

Duperier, A.: A New Cosmic-Ray Recorder and the Air Absorption and Decay of Particles, Terrest. Magn. Atmosph. Electr., 49, 1-7, 1944.

Dvornikov, V. M., Krestyannikov, Yu. Ya., and Sergeev, A. V.: Determination of the mass-average temperature on the cosmic ray intensity data, Geomagn. Aeron., 16, 923-925, 1976.

Forsythe, G. E., Malkolm, M. A., and Moler, C. B.: Computer methods for mathematical computations: Mir, Moscow, USSR, 1980. 
Maeda, K. and Wada, M.: Atmospheric temperature effect upon the cosmic ray intensity at sea level, J. Sci. Res. Inst., Tokyo, 48, 71-79, 1954.

Okazaki, Y., Fushishita, A., Narumi, T., Kato, C., Yasue, S., Kuwabara, T., Bieber, J. W., Evenson, P., Da Silva, M. R., Dal Lago, A., Schuch, N. J., Fujii, Z., Duldig, M. L., Humble, J. E., Sabbah, I., Kota, J., and Munakata, K.: Drift effects and the cosmic ray density gradient in a solar rotation period: First observation with the Global Muon Detector Network (GMDN), Astrophys. J., 681, 693-707, 2008.
Tilav, S., Desiati, P., Kuwabara, T., Rocco, D., Rothmaier, F., Simmons, M., Wissing, H., for the IceCube Collaboration: Atmospheric Variations as observed by IceCube, in: Proc. $31^{s t}$ ICRC, Lodz, Poland, 7-15 July 2009.

Yanchukovsky, V., Filimonov, G., and Hisamov, G.: Atmospheric Variations in Muon Intensity for Different Zenith Angles, Bull. Russ. Acad. Sci.: Physics, 71, 1038-1040, 2007. 